\title{
Measure rigidity and $p$-adic Littlewood-type problems
}

\author{
Manfred Einsiedler and Dmitry Kleinbock
}

\begin{abstract}
Various $p$-adic versions of Littlewood's conjecture are investigated, generalizing a set-up considered recently by de Mathan and Teulié. In many cases it is shown that the sets of exceptions to these conjectures have Hausdorff dimension zero. The proof follows the measure ridigity approach of Einsiedler, Katok and Lindenstrauss.
\end{abstract}

\section{Introduction}

\subsection{The conjecture and our main theorems}

Every real number $u$ satisfies

$$
\left|q u-q_{0}\right|<\frac{1}{q} \quad \text { for infinitely many pairs }\left(q, q_{0}\right) \in \mathbb{Z}^{2}
$$

(where $q \neq 0$ is understood). Here the exponent of $q$ is sharp in the sense that for certain (badly approximable) numbers, e.g. for irrational elements of a quadratic number field, there exists a constant $\epsilon>0$ such that

$$
\left|q u-q_{0}\right| \geqslant \frac{\epsilon}{q} \text { for all pairs }\left(q, q_{0}\right) \in \mathbb{Z}^{2}
$$

or, equivalently,

$$
\liminf _{q \rightarrow \infty}|q| \cdot\langle q u\rangle>0
$$

where $\langle z\rangle \stackrel{\text { def }}{=} \min _{q_{0} \in \mathbb{Z}}\left|z-q_{0}\right|$ denotes the distance to the nearest integer.

In the following let $p$ be a prime number. De Mathan and Teulié [DT04] conjectured that for every $u \in \mathbb{R}$ and $\epsilon>0$

$$
\left|q u-q_{0}\right|<\frac{\epsilon}{q|q|_{p}}=\frac{\epsilon}{q^{\prime}} \quad \text { for infinitely many pairs }\left(q, q_{0}\right) \in \mathbb{Z}^{2},
$$

where $q=q^{\prime} p^{k}$ for some $k \geqslant 0, q^{\prime}$ is coprime to $p$, and $|q|_{p}=1 / p^{k}$ denotes the $p$-adic norm. Equivalently, one can ask whether

$$
\liminf _{q \rightarrow \infty}|q| \cdot|q|_{p} \cdot\langle q u\rangle=0
$$

or (see [DT04, Lemme 1.3]) whether the digits in the continued fraction expansions for $u, p u, p^{2} u, \ldots$ do not all allow a uniform bound. Moreover, de Mathan and Teulié showed that this property holds for elements of a quadratic number field.

We show in the present paper that the above property holds except possibly for a set of Hausdorff dimension zero.

Received 26 October 2005, accepted in final form 18 December 2006.

2000 Mathematics Subject Classification 11J04 (primary), 11J83, 37D40, 37A35 (secondary).

Keywords: invariant measures, rigidity, higher rank abelian actions, Diophantine approximation, p-adic, Littlewood's conjecture.

This journal is (C) Foundation Compositio Mathematica 2007. 


\section{Einsiedler And D. Kleinbock}

Theorem 1.1. The set of $u \in \mathbb{R}$ which do not satisfy (1.1) has Hausdorff dimension zero; in fact, it is a countable union of sets with box dimension zero.

The above theorem is derived from the following result.

Theorem 1.2. For any $v \in \mathbb{Q}_{p}$, the set of $u \in \mathbb{R}$ which do not satisfy

$$
\liminf _{q \rightarrow \infty, q_{0} \rightarrow \infty}|q| \cdot\left|q u-q_{0}\right| \cdot\left|q v-q_{0}\right|_{p}=0
$$

has Hausdorff dimension zero; in fact, it is a countable union of sets with box dimension zero.

It is easy to see that Theorem 1.2 implies Theorem 1.1. Indeed, if $u \neq 0$ satisfies (1.2) with $v=0$, then, dividing by $u$ and interchanging $q$ and $q_{0}$, one obtains

$$
\liminf _{q \rightarrow \infty, q_{0} \rightarrow \infty}\left|q_{0}\right| \cdot\left|\frac{q}{u}-q_{0}\right| \cdot|q|_{p}=0 .
$$

We claim that when $q_{0} \neq 0$ and the above product is small enough, $|q / u|$ must be smaller than $2\left|q_{0}\right|$ : otherwise

$$
\left|q_{0}\right| \cdot\left|\frac{q}{u}-q_{0}\right| \cdot|q|_{p} \geqslant\left|\frac{q}{u}-q_{0}\right| \cdot|q|_{p} \geqslant \frac{1}{2}\left|\frac{q}{u}\right| \cdot|q|_{p} \geqslant \frac{1}{2|u|},
$$

contradicting (1.3). Hence, (1.1) holds with $u$ replaced by $1 / u$.

An assertion that is stronger than Theorem 1.2 , namely that the set of pairs $(u, v) \in \mathbb{R} \times \mathbb{Q}_{p}$ which do not satisfy (1.2) is a countable union of sets with box dimension zero, appears to be within reach, and will be the subject of a forthcoming paper (see $\S 5.4$ ). It seems natural to conjecture, generalizing the conjecture of de Mathan and Teulié, that (1.2) holds for any $(u, v) \in \mathbb{R} \times \mathbb{Q}_{p}$ except for $u=0, v=0$.

\subsection{Connection to Littlewood's conjecture}

The above conjecture, as well as its weaker version due to de Mathan and Teulié, look very similar to Littlewood's conjecture (circa 1930) which states

$$
\liminf _{q \rightarrow \infty} q\langle q u\rangle\langle q v\rangle=0
$$

for every $(u, v) \in \mathbb{R}^{2}$. However, this resemblance goes deeper. For example, the result by de Mathan and Teulié on quadratic numbers [DT04] is an analogue of a result by Cassels and SwinnertonDyer [CS55] that showed (1.4) for elements of the same cubic number field (while the quadratic case is easy in the case of (1.4)).

More recently, the first named author, Katok and Lindenstrauss [EKL06] used dynamics on $\mathrm{SL}(3, \mathbb{R}) / \mathrm{SL}(3, \mathbb{Z})$ to show that Littlewood's conjecture fails at most on a set of Hausdorff dimension zero. Here the action is the $\mathbb{R}^{2}$-flow realized as left multiplication by positive diagonal matrices, i.e. $\mathbf{t}=\left(t_{1}, t_{2}\right) \in \mathbb{R}^{2}$ acts as

$$
\alpha^{\mathbf{t}}(x)=\left(\begin{array}{ccc}
e^{-\left(t_{1}+t_{2}\right)} & & \\
& e^{t_{1}} & \\
& & e^{t_{2}}
\end{array}\right) x \quad \text { for every } x \in \operatorname{SL}(3, \mathbb{R}) / \operatorname{SL}(3, \mathbb{Z}) .
$$

The proof in [EKL06] can be roughly divided into three main steps.

(i) Linking the Diophantine conjecture with the dynamics of $\alpha$.

(ii) Studying (and, if possible, classifying) $\alpha$-invariant and ergodic probability measures on $\mathrm{SL}(3, \mathbb{R}) / \mathrm{SL}(3, \mathbb{Z})$.

(iii) Linking the box dimension of an exceptional set with an $\alpha$-invariant measure of positive entropy. 
Step (i) uses at its heart the following characterization (see [Mar97, § 2], [Sta00, § 30.3] or [EKL06, Proposition 12.1]): $(u, v) \in \mathbb{R}^{2}$ fails Littlewood's conjecture (1.4) if and only if

$$
\left(\begin{array}{lll}
1 & & \\
u & 1 & \\
v & & 1
\end{array}\right) \mathrm{SL}(3, \mathbb{Z}) \text { has bounded orbit under the semigroup }\left\{\alpha^{\mathbf{t}}: \mathbf{t} \in \mathbb{R}_{+}^{2}\right\} .
$$

In principle, this could be used to solve Littlewood's conjecture if the dynamics of $\alpha$ were completely understood. Margulis [Mar00] conjectured that there are very few $\alpha$-invariant measures as in step (ii): a phenomenon called measure rigidity. However, the only currently available method to study these measures works exclusively for measures satisfying an additional assumption, namely for measures $\mu$ such that for some element $\alpha^{\mathrm{t}}$ of the action, its measure-theoretic entropy $\mathrm{h}_{\mu}\left(\alpha^{\mathrm{t}}\right)$ is positive. The complete answer for positive entropy measures was given in [EKL06, Theorem 1.3] where the two separately developed methods from [EK03] and [Lin06] were combined. In step (iii) the positive entropy assumption is then translated to the statement about the Hausdorff dimension.

\subsection{Method of proof}

For the proof of Theorem 1.2 we follow, in principle, the same scheme as described in $\S 1.2$, but use dynamics on

$$
X=G / \Gamma, \quad \text { where } G=\operatorname{SL}(2, \mathbb{R}) \times \operatorname{SL}\left(2, \mathbb{Q}_{p}\right), \Gamma=\imath\left(\operatorname{SL}\left(2, \mathbb{Z}\left[\frac{1}{p}\right]\right)\right)
$$

instead. Here $\imath: \operatorname{SL}\left(2, \mathbb{Z}\left[\frac{1}{p}\right]\right) \rightarrow G$ is the diagonal embedding, i.e. $\imath(A)=(A, A)$ for any $A \in$ $\mathrm{SL}\left(2, \mathbb{Z}\left[\frac{1}{p}\right]\right)$. The action considered is the $(\mathbb{R} \times \mathbb{Z})$-action defined by left multiplication of $x \in X$ by

$$
\alpha^{(t, n)} \stackrel{\text { def }}{=}\left(\left(\begin{array}{cc}
e^{-t} & \\
& e^{t}
\end{array}\right),\left(\begin{array}{ll}
p^{n} & \\
& p^{-n}
\end{array}\right)\right) \quad \text { for }(t, n) \in \mathbb{R} \times \mathbb{Z} .
$$

We prove in $\S 2$ (see Proposition 2.2) that $u \in \mathbb{R} \backslash\{0\}$ and $v \in \mathbb{Z}_{p}$ fail (1.2) if and only if

$$
x_{u, v} \stackrel{\text { def }}{=}\left(\left(\begin{array}{ll}
1 & \\
u & 1
\end{array}\right),\left(\begin{array}{ll}
1 & \\
v & 1
\end{array}\right)\right) \Gamma
$$

has bounded orbit under the semigroup $\alpha \stackrel{\text { def }}{=}\left\{\alpha^{(t, n)}:(t, n) \in C\right\}$, where

$$
C=\{(t, n) \in \mathbb{R} \times \mathbb{Z}: n \geqslant 0, t-n \log p \geqslant 0\} .
$$

This serves as a replacement for part (i) of the argument of [EKL06], and sets the stage for part (ii), that is, using measure rigidity methods. Namely, applying a recent result by Lindenstrauss [Lin06, Theorem 1.1] (see Theorem 3.1), we prove that any $\alpha$-invariant and ergodic probability measure on $X$ for which the measure-theoretic entropy (see $\S 3$ ) with respect to $\alpha^{(1,0)}$ is positive has to be the unique $G$-invariant Haar measure on $X$. Consequently, no $\alpha$-invariant and ergodic probability measure supported on a compact subset $Y$ of $X$ has positive entropy for $\alpha^{(1,0)}$.

From this, using a generalization of step (iii) of the proof in [EKL06] (see Proposition 4.1), we derive that for any compact subset $B$ of the unstable horospherical subgroup relative to $\alpha^{(1,0)}$ (defined in $\S 4$ ), any compact $Y \subset X$ and any $x \in X$, the set

$$
\left\{h \in B: \alpha^{C} h x \subset Y\right\}
$$

has box dimension zero. To finish the proof of Theorem 1.2 it remains to observe that $\left\{x_{u, v}: u \in \mathbb{R}\right\}$ is precisely the orbit of $x_{0, v}$ under the unstable horospherical subgroup relative to $\alpha^{(1,0)}$.

This paper is organized as follows. The aforementioned three parts of the argument are discussed in detail in $\S \S 2-4$, respectively, and then used in $\S 5.1$ where we finish the proof of Theorem 1.2. After that, we give several generalizations and open questions. In particular, we point out that, 


\section{Einsiedler AND D. Kleinbock}

while Littlewood's conjecture does not seem to become easier to prove in higher dimensions, the following modification of the de Mathan-Teulié conjecture follows quite easily from a theorem of Furstenberg [Fur67].

Theorem 1.3. Let $p_{1}, p_{2}$ be two distinct prime numbers. Then for every $u \in \mathbb{R}$

$$
\liminf _{q \rightarrow \infty}|q| \cdot|q|_{p_{1}} \cdot|q|_{p_{2}} \cdot\langle q u\rangle=0 .
$$

This theorem is proved in $\S 5.2$. Another modification of (1.1), where the $p$-adic norm is replaced by a 'pseudo-norm'

$$
|q|_{\mathcal{D}}=\inf \left\{1 / r_{n}: q \in r_{n} \mathbb{Z}\right\},
$$

is discussed in $\S 5.3$. Here $\mathcal{D}=\left(r_{n}\right)_{n \in \mathbb{Z}_{+}}$is a sequence of positive integers with $r_{0}=1$ and $r_{n+1} \in r_{n} \mathbb{Z}$ for each $n$; the choice $r_{n}=p^{n}$ gives the usual $p$-adic norm. De Mathan and Teulié proved in [DT04] that every quadratic irrational $u$ satisfies $\lim \inf _{q \rightarrow \infty}|q| \cdot|q|_{\mathcal{D}} \cdot\langle q u\rangle=0$ for any $\mathcal{D}$ as above with uniformly bounded ratios $r_{n+1} / r_{n}$. In this paper we explain how a modification of our method yields a $\mathcal{D}$-adic analogue of Theorem 1.2 in the case $\mathcal{D}=\left(a^{n}\right)_{n \in \mathbb{N}}$, where $a$ is not necessarily prime.

\section{Dynamical characterization of conditions (1.1) and (1.2)}

\subsection{Lattices in $\mathbb{R}^{2} \times \mathbb{Q}_{p}^{2}$ and Mahler's criterion}

In the following we assume that the metric (and the topology) of $X=G / \Gamma$ as in (1.5) is induced by a right-invariant metric on $G$.

It is well known that $\mathbb{Z}\left[\frac{1}{p}\right]^{2}$ diagonally imbedded in $\mathbb{R}^{2} \times \mathbb{Q}_{p}^{2}$ is a lattice, that is, it is discrete and the quotient $\left(\mathbb{R}^{2} \times \mathbb{Q}_{p}^{2}\right) / \mathbb{Z}\left[\frac{1}{p}\right]^{2}$ has finite volume (in fact, equal to 1 ). For any $g \in G, g \mathbb{Z}\left[\frac{1}{p}\right]^{2}$ is also a lattice in $\mathbb{R}^{2} \times \mathbb{Q}_{p}^{2}$ of covolume 1 . In other words, $g \mathbb{Z}\left[\frac{1}{p}\right]^{2}$ consists of all elements of the form

$$
\left(A\left(\begin{array}{c}
q \\
q_{0}
\end{array}\right), B\left(\begin{array}{c}
q \\
q_{0}
\end{array}\right)\right) \quad \text { for } q, q_{0} \in \mathbb{Z}\left[\frac{1}{p}\right],
$$

where $A \in \operatorname{SL}(2, \mathbb{R})$ and $B \in \mathrm{SL}\left(2, \mathbb{Q}_{p}\right)$. That is, it is a free $\mathbb{Z}\left[\frac{1}{p}\right]$-module generated by $\left(\mathbf{a}_{1}, \mathbf{b}_{1}\right)$, $\left(\mathbf{a}_{2}, \mathbf{b}_{2}\right)$, where $A$ has column vectors $\mathbf{a}_{1}, \mathbf{a}_{2} \in \mathbb{R}^{2}$ and $B$ has column vectors $\mathbf{b}_{1}, \mathbf{b}_{2} \in \mathbb{Q}_{p}^{2}$.

It is easy to see that $\Gamma$ is exactly the stablilizer of $\mathbb{Z}\left[\frac{1}{p}\right]^{2}$ under the action described above, and therefore $X=G / \Gamma$ can be identified with the $G$-orbit of $\mathbb{Z}\left[\frac{1}{p}\right]^{2}$. In other words, points $x=(A, B) \Gamma \in$ $X$ are identified with unimodular lattices $\Lambda_{x}$ in $\mathbb{R}^{2} \times \mathbb{Q}_{p}^{2}$ that are generated, as above, by the column vectors of $A$ and $B$.

This correlation between points in $X$ and certain lattices allows a convenient description of compact subsets of $X$ (see [KT, Theorem 7.10] for a more general formulation of the $S$-adic Mahler's criterion).

Theorem 2.1 (Mahler's criterion for $X$ ). A subset $L \subset X$ has compact closure if and only if there exists some $\delta>0$ so that

$$
L \subset K_{\delta} \stackrel{\text { def }}{=}\left\{x \in X: \Lambda_{x} \cap B_{\delta}^{\mathbb{R}^{2} \times \mathbb{Q}_{p}^{2}}(0)=\{0\}\right\} .
$$

Proof. The implication $(\Longrightarrow)$ is trivial (and not needed for our purposes). For the converse, we need to show that $K_{\delta}$ is compact for every positive $\delta$. Let us denote $\operatorname{SL}(2, \mathbb{R}) \times \operatorname{SL}\left(2, \mathbb{Z}_{p}\right)$ by $G^{\prime}$, and observe that $G=G^{\prime} \Gamma$ due to the strong approximation theorem [Kne65]; in other words, every $g \in G$ can be represented as $g=g_{f} g_{l}$, where $g_{f} \in G^{\prime}$ and $g_{l} \in \Gamma$. Hence,

$$
X \cong G^{\prime} /\left(G^{\prime} \cap \Gamma\right)=G^{\prime} / \operatorname{SL}(2, \mathbb{Z}) \cong\left\{g \mathbb{Z}^{2}: g \in G^{\prime}\right\}
$$


Furthermore, one has

$$
\begin{aligned}
g \mathbb{Z}\left[\frac{1}{p}\right]^{2} \cap \mathbb{R}^{2} \times \mathbb{Z}_{p}^{2} & =g_{f} \mathbb{Z}\left[\frac{1}{p}\right]^{2} \cap\left(\mathbb{R}^{2} \times \mathbb{Z}_{p}^{2}\right) \\
& =g_{f}\left(\mathbb{Z}\left[\frac{1}{p}\right]^{2} \cap\left(\mathbb{R}^{2} \times \mathbb{Z}_{p}^{2}\right)\right)=g_{f} \mathbb{Z}^{2} .
\end{aligned}
$$

Thus one can identify $K_{\delta}$ with

$$
\left\{g \mathbb{Z}^{2}: g \in G^{\prime}, g \mathbb{Z}^{2} \cap B_{\delta}^{\mathbb{R}^{2} \times \mathbb{Z}_{p}^{2}}(0)=\{0\}\right\} .
$$

To finish the proof it remains to observe that the projection $G^{\prime} \rightarrow \operatorname{SL}(2, \mathbb{R})$ induces a surjection $G / \Gamma \rightarrow \mathrm{SL}(2, \mathbb{R}) / \mathrm{SL}(2, \mathbb{Z})$ with compact fibers, and the set $(2.1)$ is contained in the preimage of

$$
\left\{g \mathbb{Z}^{2}: g \in \mathrm{SL}(2, \mathbb{R}), g \mathbb{Z}^{2} \cap B_{\delta}^{\mathbb{R}^{2}}(0)=\{0\}\right\},
$$

which is compact due to the original Mahler's criterion (see [Rag72, Corollary 10.9]).

\subsection{Theorem 1.2 and cone orbits}

A cone $C$ of $\mathbb{R} \times \mathbb{Z}$ is a subset defined by two inequalities $(t, n) \cdot \mathbf{v}_{1} \geqslant 0$ and $(t, n) \cdot \mathbf{v}_{2} \geqslant 0$ where $\mathbf{v}_{1}, \mathbf{v}_{2} \in \mathbb{R}^{2}$ are linearly independent vectors. The cone orbit of a point $x \in X$ is given by

$$
\alpha^{C} x=\left\{\alpha^{(t, n)} x:(t, n) \in C\right\},
$$

where $\alpha$ is as defined in (1.6).

Proposition 2.2. Let $C$ be as in (1.8), and let $u \in \mathbb{R}$ be nonzero. Then $(u, v)$ satisfies (1.2) if and only if the $C$-orbit of $x_{u, v}$ as in (1.7) is unbounded.

We will only prove the 'if' part, since this is the only direction that will be needed for Theorem 1.2. The converse is not difficult either.

Proof. Suppose that $x_{u, v}$ has unbounded $C$-orbit. By Mahler's criterion (Theorem 2.1) there exists, for every $\delta>0$, a pair $(t, n)$ with $n \geqslant 0$ and $e^{t} p^{-n} \geqslant 1$ such that $\Lambda_{\alpha^{(t, n)} x_{u, v}}$ contains a nonzero element in $B_{\delta}^{\mathbb{R}^{2} \times \mathbb{Q}_{p}^{2}}(0)$. Clearly, $\Lambda_{\alpha}^{(t, n)} x_{u, v}$ is generated by

$$
\left(\left(\begin{array}{c}
e^{-t} \\
e^{t} u
\end{array}\right),\left(\begin{array}{c}
p^{n} \\
p^{-n} v
\end{array}\right)\right) \text { and }\left(\left(\begin{array}{c}
0 \\
e^{t}
\end{array}\right),\left(\begin{array}{c}
0 \\
p^{-n}
\end{array}\right)\right) .
$$

However, since $\Lambda_{\alpha^{(t, n)} x_{u, v}}$ is a $\mathbb{Z}\left[\frac{1}{p}\right]$-module, the vectors

$$
\left(\left(\begin{array}{c}
e^{-t} p^{-n} \\
e^{t} p^{-n} u
\end{array}\right),\left(\begin{array}{c}
1 \\
p^{-2 n} v
\end{array}\right)\right) \text { and }\left(\left(\begin{array}{c}
0 \\
e^{t} p^{-n}
\end{array}\right),\left(\begin{array}{c}
0 \\
p^{-2 n}
\end{array}\right)\right)
$$

are also generators. Therefore, there exists some nonzero $\left(q, q_{0}\right) \in \mathbb{Z}\left[\frac{1}{p}\right]^{2}$ such that

$$
\left(\left(\begin{array}{c}
e^{-t} p^{-n} q \\
e^{t} p^{-n}\left(q u-q_{0}\right)
\end{array}\right),\left(\begin{array}{c}
q \\
p^{-2 n}\left(q v-q_{0}\right)
\end{array}\right)\right) \in \mathbb{R}^{2} \times \mathbb{Q}_{p}^{2}
$$

is $\delta$-small. In particular, $|q|_{p}$ is less than $\delta$, which (assuming $\delta \leqslant 1$ ) implies that $q \in \mathbb{Z}$. Since $n \geqslant 0$ and $v \in \mathbb{Z}_{p}$, the inequality

$$
p^{2 n}\left|\left(q v-q_{0}\right)\right|_{p}=\left|p^{-2 n}\left(q v-q_{0}\right)\right|_{p}<\delta
$$

shows that $q_{0} \in \mathbb{Z}$ as well.

Now consider two cases. If $u \in \mathbb{Q}$, that is, $q u-q_{0}=0$ for some $q, q_{0} \in \mathbb{Z} \backslash\{0\},(1.2)$ is obviously satisfied (simply replace $q, q_{0}$ in $(1.2)$ by $k q, k q_{0}, k \in \mathbb{N}$ ). If $u \notin \mathbb{Q}$, we use the assumption $e^{t} p^{-n} \geqslant 1$ 


\section{EinsiedleR AND D. KLEINBOCK}

to conclude that the inequality

$$
\left|e^{t} p^{-n}\left(q u-q_{0}\right)\right|<\delta
$$

can only hold for $|q|$ and $\left|q_{0}\right|$ not less than some $Q=Q(u, \delta)$, where the latter quantity tends to infinity as $\delta \rightarrow 0$. By taking the product of the inequality $\left|e^{-t} p^{-n} q\right|<\delta$ with (2.3) and (2.2), we arrive at $|q| \cdot\left|q u-q_{0}\right| \cdot\left|q v-q_{0}\right|_{p}<\delta^{3}$. Since this holds for any $\delta$, we conclude that (1.2) holds.

\subsection{The de Mathan-Teulié Conjecture and cone orbits}

Even though we were able to reduce Theorem 1.1 to Theorem 1.2, it is instructive to observe that condition (1.1) can also be characterized in a dynamical language.

Proposition 2.3. Let $C^{\prime}=\{(t, n) \in \mathbb{R} \times \mathbb{Z}: n \leqslant 0, t+n \log p \geqslant 0\}$. Then $u \in \mathbb{R} \backslash\{0\}$ satisfies (1.1) if (and only if) $x_{u, 0}$ has unbounded $C^{\prime}$-orbit, i.e. $\alpha^{C^{\prime}} x_{u, 0} \subset X$ does not have compact closure.

Proof. We again only prove the if part. As in the proof of Proposition 2.2 we find for an arbitrary $\delta>0$ some $(t, n) \in C^{\prime}$ such that $\Lambda_{\alpha^{(t, n)} x_{u, 0}}$ contains some nonzero element of $B_{\delta}^{\mathbb{R}^{2} \times \mathbb{Q}_{p}^{2}}(0)$. We multiply the obvious generators of $\Lambda_{\alpha^{(t, n)} x_{u, 0}}$ by $p^{n}$ and obtain the two generators

$$
\left(\left(\begin{array}{c}
e^{-t} p^{n} \\
e^{t} p^{n} u
\end{array}\right),\left(\begin{array}{c}
p^{2 n} \\
0
\end{array}\right)\right) \text { and }\left(\left(\begin{array}{c}
0 \\
e^{t} p^{n}
\end{array}\right),\left(\begin{array}{l}
0 \\
1
\end{array}\right)\right) \text {. }
$$

Therefore, the small element has the form

$$
\left(\left(\begin{array}{c}
e^{-t} p^{n} q \\
e^{t} p^{n}\left(q u-q_{0}\right)
\end{array}\right),\left(\begin{array}{c}
p^{2 n} q \\
-q_{0}
\end{array}\right)\right) \in \mathbb{R}^{2} \times \mathbb{Q}_{p}^{2}
$$

for some $\left(q, q_{0}\right) \in \mathbb{Z}\left[\frac{1}{p}\right]^{2}$. Since $n \leqslant 0$ we conclude from the $p$-adic part of that vector that $q, q_{0} \in \mathbb{Z}$, and in fact

$$
p^{-2 n}|q|_{p}<\delta .
$$

By definition of $C^{\prime}$ we have $e^{t} p^{n} \geqslant 1$, thus, in the case $u \notin \mathbb{Q}$ (the rational case is again easy) the inequality

$$
\left|e^{t} p^{n}\left(q u-q_{0}\right)\right|<\delta
$$

forces $|q|$ and $\left|q_{0}\right|$ to be large enough. By taking the product of the inequality $\left|e^{-t} p^{n} q\right|<\delta$ with (2.4) and (2.5), we arrive at $|q| \cdot|q|_{p} \cdot\left|q u-q_{0}\right|<\delta^{3}$.

\section{Measure rigidity on $X$}

Measure rigidity refers to the phenomenon that certain actions have very few invariant measures. The case of unipotent actions is well understood due to Ratner's work [Rat91], and extensions by Ratner [Rat95] and Margulis-Tomanov [MT94]. The case of higher rank partially hyperbolic actions, as we are concerned with here, has recently seen an interesting development [EK03, EKL06, KK01, KS05, Lin06]. However, here our understanding is not complete, and positive entropy is, so far, a crucial additional assumption. Even so, it is possible to apply these results, in particular to number theory.

Let $H$ be a locally compact metric group acting on a locally compact metric space $X$. A measure $\mu$ on $X$ is $H$-invariant if $\mu\left(h^{-1} B\right)=\mu(B)$ for any measurable $B \subset X$. A probability measure $\mu$ is $H$-ergodic if any invariant measurable set, that is any measurable $B \subset X$ with $\mu(B \Delta h B)=0$ for any $h \in H$, satisfies $\mu(B) \in\{0,1\}$. We only consider probability measures. Then the $H$-invariant and ergodic measures are the extremal points of the convex set of all $H$-invariant measures. Moreover, every $H$-invariant measure $\nu$ can be expressed as a generalized convex combination

$$
\nu=\int_{R} \mu_{r} d \rho(r)
$$


of $H$-invariant and ergodic measures $\mu_{r}$ on $X(r \in R)$, where $(R, \rho)$ is some probability space. This is the ergodic decomposition of $\mu$ with respect to $H$, and the measures $\mu_{r}$ are the ergodic components [Var63].

We will need the notion of measure theoretic entropy. Let $T: X \rightarrow X$ be a measure-preserving map on the probability space $(X, \mu)$. Instead of giving the formal definition [Wal82, ch. 4], let us just mention that the measure theoretic entropy $\mathrm{h}_{\mu}(T)$ is a non-negative (in general, possibly infinite) number that measures the complexity of the dynamical system defined by $T$ on $(X, \mu)$.

As mentioned in the introduction, the following theorem can be specialized to fit our needs. To avoid unnecessary complications we state a slightly simplified version.

Theorem 3.1 (Lindenstrauss [Lin06, Theorem 1.1]). Let $H=\operatorname{SL}(2, \mathbb{R})$, let $L$ be an $S$-arithmetic group and let $G=H \times L$. Take $\Gamma$ to be a discrete subgroup of $G$ (not necessarily a lattice) such that $\Gamma \cap L$ is finite. Suppose that $\mu$ is a probability measure on $X=G / \Gamma$ that is invariant under left multiplication by elements of the diagonal group $A$ in $\mathrm{SL}(2, \mathbb{R})$. Assume, furthermore, the following two conditions.

(i) All ergodic components of $\mu$ with respect to the action of $A$ have positive entropy.

(ii) The probability measure $\mu$ is L-recurrent, i.e. for a measurable $B \subset X$, almost every $x \in B$ satisfies, for every compact subset $C \subset L$, that there exists $\ell \in L \backslash C$ with $\ell x \in B$.

Then $\mu$ is a convex combination of algebraic measures invariant under $H$.

For the case $G=\mathrm{SL}(2, \mathbb{R}) \times \mathrm{SL}\left(2, \mathbb{Q}_{p}\right)$ and the lattice $\Gamma=\imath\left(\mathrm{SL}\left(2, \mathbb{Z}\left[\frac{1}{p}\right]\right)\right)$ we obtain the following corollary.

Corollary 3.2. Let $X=G / \Gamma$ be as in (1.5), and let $\alpha$ be the $(\mathbb{R} \times \mathbb{Z})$-action defined in (1.6). Let $\mu$ be an $\alpha$-invariant and ergodic probability measure on $X$. If $\mathrm{h}_{\mu}\left(\alpha^{(1,0)}\right)>0$, then $\mu$ is the unique $\mathrm{SL}(2, \mathbb{R}) \times \mathrm{SL}\left(2, \mathbb{Q}_{p}\right)$-invariant Haar measure $\mu=m_{X}$ of $X$.

The following general property of entropy will be useful (see [Wal82, Theorem 8.2] in the case of the ergodic decomposition and [Jac63] for the general case). If $T: X \rightarrow X$ is continuous, $\nu$ is $T$-invariant and $\nu=\int_{R} \mu_{r} d \rho(r)$ is a convex combination of other $T$-invariant probability measures, then

$$
\mathrm{h}_{\nu}(T)=\int \mathrm{h}_{\mu_{r}}(T) d \rho(r) .
$$

In particular, the entropy $\mathrm{h}_{\nu}(T)$ for a non-ergodic measure $\nu$ as in (3.1) can be calculated from the entropy $\mathrm{h}_{\mu_{r}}(T)$ of the ergodic components $\mu_{r}$ (and the measure $\rho$ ).

Proof. We will apply Theorem 3.1 to $L=\mathrm{SL}\left(2, \mathbb{Q}_{p}\right)$ and $\Gamma$ as above. Clearly, $\Gamma \cap L$ is the trivial group. Now let $\mu$ be an $\alpha$-invariant and ergodic probability measure on $X$. Then it is invariant under $A$. Note, however, that $\mu$ might not be ergodic under $A$. So, by the ergodic decomposition, $\mu$ possibly decomposes in a generalized convex combination $\mu=\int_{R} \nu_{r} d \rho$ of $A$-ergodic measures $\nu_{r}$, where $(R, \rho)$ is some probability space. However, ergodicity of $\mu$ under $\alpha$ implies that $\mathrm{h}_{\nu_{r}}\left(\alpha^{(1,0)}\right)=\mathrm{h}_{\mu}\left(\alpha^{(1,0)}\right)$ for $\rho$-almost every $r \in R$. By assumption, this entropy is positive, so condition (1) in Theorem 3.1 is satisfied.

Since $\mu$ is a probability measure and invariant under $\alpha^{(0,1)}$, it is also recurrent under $\alpha^{(0,1)}$ by Poincaré recurrence. Since $\alpha^{(0,1)}$ is left multiplication by an element of $L$ (whose powers are not contained in a compact subset of $L$ ), it follows that $\mu$ is recurrent under $L$ as required in condition (2).

Therefore, $\mu$ is a (generalized) convex combination of homogeneous measures that are invariant under $\mathrm{SL}(2, \mathbb{R})$ by Theorem 3.1. The corollary follows from the following proposition. 


\section{EinsiedleR AND D. Kleinbock}

Proposition 3.3. Let $X=G / \Gamma$ be as in (1.5). The Haar measure $m_{X}$ is the only homogeneous measure $\nu$ on $X$ that is invariant under $\mathrm{SL}(2, \mathbb{R})$.

Proof. The proposition follows from [Tom00, Theorem 1] since $\Gamma$ is an $S$-arithmetic lattice, and since no proper finite index subgroup of $G$ exists. We also give a short self-contained proof.

Let $H^{\prime} \subset G$ be a closed subgroup that contains $\operatorname{SL}(2, \mathbb{R})$ such that $\nu$ is the Haar measure on a single closed $H^{\prime}$-orbit $H^{\prime} x$. Let $x=g \Gamma$ for some $g=\left(g_{\infty}, g_{p}\right) \in G$, then $H^{\prime} x \simeq H^{\prime} / \Lambda^{\prime}$ where $\Lambda^{\prime}=g \Gamma g^{-1} \cap H^{\prime}$ is a lattice in $H^{\prime}$. Clearly, $H^{\prime \prime}=H^{\prime} \cap\left(\mathrm{SL}(2, \mathbb{R}) \times \mathrm{SL}\left(2, \mathbb{Z}_{p}\right)\right)$ is an open closed subgroup of $H^{\prime}$. Therefore, $\Lambda^{\prime \prime}=\Lambda^{\prime} \cap H^{\prime \prime}$ is a lattice in $H^{\prime \prime}$, and the factor map $\pi: H^{\prime \prime} \rightarrow \operatorname{SL}(2, \mathbb{R})$ with compact kernel $H^{\prime \prime} \cap \mathrm{SL}\left(2, \mathbb{Z}_{p}\right)$ descends to a factor map $\pi: H^{\prime \prime} / \Lambda^{\prime} \rightarrow \operatorname{SL}(2, \mathbb{R}) / \pi\left(\Lambda^{\prime \prime}\right)$. It follows that $\pi\left(\Lambda^{\prime \prime}\right) \subset \mathrm{SL}(2, \mathbb{R})$ is a lattice. However, since $\Gamma_{g}=\pi\left(g \Gamma g^{-1} \cap\left(\mathrm{SL}(2, \mathbb{R}) \times \mathrm{SL}\left(2, \mathbb{Z}_{p}\right)\right)\right)$ is also a lattice (that is commensurable to $\left.g_{\infty} \mathrm{SL}(2, \mathbb{Z}) g_{\infty}^{-1}\right)$, it follows that $\pi\left(\Lambda^{\prime \prime}\right) \subset \Gamma_{g}$ has finite index. Let

$$
u_{N}=\left(\begin{array}{cc}
1 & N \\
& 1
\end{array}\right)
$$

be an integer matrix (which we can consider as an element of $\mathrm{SL}(2, \mathbb{R})$ as well as $\mathrm{SL}\left(2, \mathbb{Q}_{p}\right)$ ), and let $u_{N}^{\mathrm{T}}$ denote its transpose. Then for sufficiently large $N$ we have $g_{\infty} u_{N} g_{\infty}^{-1}, g_{\infty} u_{N}^{\mathrm{T}} g_{\infty}^{-1} \in \pi\left(\Lambda^{\prime \prime}\right)$, and so by the definition of $\Gamma$ we have $g_{p} u_{N} g_{p}^{-1}, g_{p} u_{N}^{\mathrm{T}} g_{p}^{-1} \in H^{\prime \prime}$. These two elements generate a subgroup whose closure is $g_{p} K_{n} g_{p}^{-1}$ for some $n$, where

$$
K_{n}=\left\{(\mathrm{Id}, A): A \in \mathrm{SL}\left(2, \mathbb{Z}_{p}\right), A \equiv \mathrm{Id}\left(\bmod p^{n}\right)\right\} .
$$

We conclude that $H^{\prime}$ contains $K_{m}$ for some $m$. Using the definition of $\Gamma$ it follows that $H^{\prime} x=X$ and that the unique $H^{\prime}$-invariant measure $\nu$ on $X$ is the Haar measure of $X$.

\section{The box dimension for points with cone orbit inside a compact set}

In the case of a single continuous map $T: X \rightarrow X$ on a compact metric space, it is well known that every orbit is equidistributed (with respect to $\mu$ ) if and only if $\mu$ is the only invariant probability measure on $X$. In the case of a statement such as Theorem 3.1 we cannot make a statement about every orbit; we can, however, deduce that the set of points with bounded orbits has small dimension.

We will give the result in a more general setting than is used in this paper. For this let $G=$ $\prod_{\sigma \in S} G_{\sigma}$ be a product of real and $p$-adic Lie groups $G_{\sigma}$, where $S \subset\{\infty, p: p \in \mathbb{N}$ is prime $\}$ is a finite set of places of $\mathbb{Q}$, let $\Gamma \subset G$ be a discrete subgroup, and let $\alpha: \mathbb{R}^{k} \times \mathbb{Z}^{\ell} \rightarrow G$ be a homomorphism such that its image consists of semisimple elements of $G$. Then we consider $\alpha$ as an $\mathbb{R}^{k} \times \mathbb{Z}^{\ell}$-action on $X=G / \Gamma$. Let $d(\cdot, \cdot)$ be a right invariant metric on $G$ which also induces a metric on $X$, again denoted by $d(\cdot, \cdot)$. We assume that the metric $d$ is such that the locally defined maps log and exp between $G$ and its Lie algebra $\mathfrak{g}$ are Lipshitz. Here the Lie algebra is the product of the real and $p$-adic Lie algebras $\mathfrak{g}_{\sigma}$ corresponding to the factors $G_{\sigma}$ for $\sigma \in S$, and we equip it with the metric derived from norms on the real and $p$-adic Lie algebras $\mathfrak{g}_{\sigma}$. For a subgroup $H \subset G$ we write $B_{r}^{H}(e)$ for the $r$-ball around the identity in $H$, where we use the restriction of the metric $d$ to $H$.

For a fixed $(\mathbf{t}, \mathbf{n}) \in \mathbb{R}^{k} \times \mathbb{Z}^{\ell}$ we let

$$
U^{+}=\left\{g \in G: \alpha^{j(\mathbf{t}, \mathbf{n})} g \alpha^{-j(\mathbf{t}, \mathbf{n})} \rightarrow e \text { for } j \rightarrow-\infty\right\}
$$

be the horospherical unstable subgroup corresponding to $\alpha^{(\mathbf{t}, \mathbf{n})}$; the horospherical stable subgroup is defined similarly. In a sense, the orbits of $U^{+} x$ are the unstable 'manifolds' (in the presence of $p$-adic Lie groups it is not a manifold in the usual sense) for $\alpha^{(\mathbf{t}, \mathbf{n})}$ through $x \in X$.

A subset $C \subset \mathbb{R}^{k} \times \mathbb{Z}^{\ell}$ defined by $C=\left\{(\mathbf{t}, \mathbf{n}):(\mathbf{t}, \mathbf{n}) \cdot \mathbf{v}_{i} \geqslant 0\right\}$, where $\mathbf{v}_{i} \in \mathbb{R}^{k+\ell}$ are given vectors for $i=1, \ldots, I$, is a cone if the group generated by $C$ is $\mathbb{R}^{k} \times \mathbb{Z}^{\ell}$. We are interested in the parts of the orbits corresponding to $C$. Here we allow $I=0$ and $C=\mathbb{R}^{k} \times \mathbb{Z}^{\ell}$. 
Before we state the main result proved in this section, let us recall the definition of the box dimension of a subset $E$ of a metric space $(Z, d)$. For $\delta>0$, let $s_{\delta}(E)$ be the maximal number of points $u_{1}, u_{2}, \ldots$ such that $d\left(u_{i}, u_{j}\right)>\delta$ for any $i \neq j$, then the (upper) box dimension is

$$
\operatorname{dim}_{\text {box }}(E)=\limsup _{\delta \rightarrow 0} \frac{\log s_{\delta}(E)}{|\log \delta|} .
$$

Proposition 4.1. Suppose that $\alpha$ and $X=G / \Gamma$ are as above, and that $C \subset \mathbb{R}^{k} \times \mathbb{Z}^{\ell}$ is a cone that contains $(\mathbf{t}, \mathbf{n})$. Let $Y \subset X$ be a compact set such that no $\alpha$-invariant and ergodic probability measure supported on $Y$ has positive entropy for $\alpha^{(\mathbf{t}, \mathbf{n})}$. Then for any compact $B \subset U^{+}$of the unstable horospherical subgroup and any $x \in X$ the set

$$
E_{B, x}(Y)=\left\{u \in B: \alpha^{C} u x \subset Y\right\}
$$

has box dimension zero. Moreover, if in addition $-(\mathbf{t}, \mathbf{n}) \in C$, then the same is true for compact subsets $B \subset U^{+} U^{-}$, and the box dimension of

$$
Y_{C}=\left\{y \in Y: \alpha^{C} y \subset Y\right\}
$$

is at most $\operatorname{dim}_{\text {box }}\left(B_{1}^{G}(e)\right)-\operatorname{dim}_{\text {box }}\left(B_{1}^{U^{+}}(e)\right)-\operatorname{dim}_{\text {box }}\left(B_{1}^{U^{-}}(e)\right)$.

The proof of Proposition 4.1 closely follows [EKL06, Proposition 9.3] which gives a similar statement for $\operatorname{SL}(k, \mathbb{R}) / \operatorname{SL}(k, \mathbb{Z})$ and $k \geqslant 3$.

We recall the notion of topological entropy for a continuous transformation $\beta: Y \rightarrow Y$ on a compact metric space $(Y, d)$. For $\epsilon>0$ and a positive integer $n$, let $s_{\epsilon, n}(\beta)$ be the maximal number of points $y_{1}, y_{2}, \ldots$ such that for any $i \neq j$ there exists some $0 \leqslant k<n$ with $d\left(\beta^{k} y_{i}, \beta^{k} y_{j}\right)>\epsilon$. Then the topological entropy is defined by

$$
\mathrm{h}_{\mathrm{top}}(\beta)=\lim _{\epsilon \rightarrow 0} \limsup _{n \rightarrow \infty} \frac{\log s_{\epsilon, n}(\beta)}{n} .
$$

Lemma 4.2. Suppose that either of the statements of Proposition 4.1 were not true. Then the restriction $\beta$ of $\alpha^{(\mathbf{t}, \mathbf{n})}$ to the $\left.\alpha\right|_{C}$-invariant compact set $Y_{C}$ has positive topological entropy.

Proof. It is easy to check that $Y_{C}$ is compact and $\alpha^{(\mathbf{s}, \mathbf{m})}$-invariant for any $(\mathbf{s}, \mathbf{m}) \in C$. Also note that there exists a uniform injectivity radius $\delta>0$ such that for any $y \in Y$ the map $g \in B_{2 \delta}^{G}(e) \mapsto g y \in X$ is an isometry. For simplicity write $a=\alpha^{(\mathbf{t}, \mathbf{n})} \in G$. Recall that, by assumption, $a$ is semisimple, i.e. $\operatorname{Ad}_{a}$ restricted to $\mathfrak{g}_{\sigma}$ is diagonalizable over the algebraic closures of $\mathbb{Q}_{\sigma}$ for every $\sigma \in S$. Using the locally defined maps $\exp (\cdot)$ and $\log (\cdot)$, one sees easily that the direct factor of the Lie algebra $\mathfrak{u}^{+}$of $U^{+}$corresponding to $\sigma \in S$ is generated by the eigenspaces of the restriction of $\operatorname{Ad}_{a}$ to $\mathfrak{g}_{\sigma}$ associated with eigenvalues of absolute value larger than one. Note that if an eigenvalue $\zeta$ is not in $\mathbb{Q}_{\sigma}$, then there is a corresponding $\operatorname{Ad}_{a}$-invariant subspace of $\mathfrak{g}_{\sigma}$ isomorphic to a vector space over $\mathbb{Q}_{\sigma}[\zeta]$ such that application of $\operatorname{Ad}_{a}$ is conjugated to multiplication by $\zeta$. By a slight abuse of language, we again call this invariant subspace of $\mathfrak{g}_{\sigma}$ an eigenspace. From this description of $\mathfrak{u}^{+}$, we learn that there exists $\lambda>1$ and $c>0$ such that $\left\|\operatorname{Ad}_{a}^{j} w\right\| \geqslant c \lambda^{j}\|w\|$ for all $w \in \mathfrak{u}^{+}$and all $j \geqslant 0$, where $\|\cdot\|$ is the sum of some fixed norms on the Lie algebras $\mathfrak{u}_{\sigma}^{+}$for $\sigma \in S$ (for more details see $[$ EK05, § 4]).

Similarly, let $\mathfrak{u}^{-}$be the Lie algebra of $U^{-}$, and let $\mathfrak{u}^{0}$ be the linear hull of the eigenspaces (in the above sense) corresponding to all eigenvalues of $\operatorname{Ad}_{a}$ with absolute value one. Note that by the assumption of semisimplicity, the Lie algebra $\mathfrak{g}$ is a direct sum of $\mathfrak{u}^{+}, \mathfrak{u}^{-}$and $\mathfrak{u}^{0}$. Also note that $\exp \left(\operatorname{Ad}_{a} u\right)=a \exp (u) a^{-1}$ for $u \in \mathfrak{g}\left(\right.$ where $\exp \left(\left(u_{\sigma}\right)_{\sigma \in S}\right)=\left(\exp \left(u_{\sigma}\right)\right)_{\sigma \in S}$ is defined coordinatewise for small enough $\left.u=\left(u_{\sigma}\right)_{\sigma \in S}\right)$.

Recall our assumption that the locally defined maps exp and log are Lipshitz. Therefore, we conclude that there exists some $\epsilon>0$ such that for $u, v \in U^{+}$with $\lambda^{-n}<d(u, v)<\delta$, one can 


\section{EinsiedleR AND D. Kleinbock}

find $j$ with $0 \leqslant j<n$ and $\epsilon<d\left(a^{j} u, a^{j} v\right)=d\left(a^{j} u a^{-j}, a^{j} v a^{-j}\right)<\delta$. The same holds similarly for $u, v \in U^{-}$and negative values of $j$.

We first consider the statement about $E_{B, x}=E_{B, x}(Y)$ in Proposition 4.1 where $B=B_{\delta / 2}^{U^{+}}(e) \subset$ $U^{+}$and $x \in X$. Denote by $\beta$ the restriction of multiplication by $a$ to $Y_{C}$. Then the above shows that for any two points $u, v \in E_{B, x}$ with $d(u, v)>\lambda^{-n}$, there exists a $j$ with $0 \leqslant j<n$ such that $d\left(\beta^{j} u x, \beta^{j} v x\right)>\epsilon$, where we used the fact that $\delta$ is an injectivity radius for $Y$. Note that by the definition of $E_{B, x}$ we have $\beta^{j} u x, \beta^{j} v x \in Y$ for all $j \geqslant 0$. Therefore, $s_{\epsilon, n}(\beta) \geqslant s_{\lambda^{-n}}\left(E_{B, x}\right)$. With this it is easy to derive the lemma in this case. In fact,

$$
\begin{aligned}
\mathrm{h}_{\text {top }}(\beta) & \geqslant \limsup _{n \rightarrow \infty} \frac{\log s_{\epsilon, n}(\beta)}{n} \geqslant(\log \lambda) \limsup _{n \rightarrow \infty} \frac{\log s_{\lambda^{-n}}\left(E_{B, x} x\right)}{\left|\log \lambda^{-n}\right|} \\
& =(\log \lambda) \limsup _{\delta \rightarrow 0} \frac{\log s_{\delta}\left(E_{B, x} x\right)}{|\log \delta|}=(\log \lambda) \operatorname{dim}_{\text {box }}\left(E_{B, x} x\right)>0,
\end{aligned}
$$

where, passing from the first line to the second line, we used the fact that for any sequence $\delta_{j} \rightarrow 0$ one can choose a sequence $n_{j} \in \mathbb{N}$ such that $\log \lambda^{-n_{j}} / \log \delta_{j} \rightarrow 1$. In general, $B \subset U^{+}$is assumed to be compact, and therefore can be covered by balls as above. This shows the lemma in the case of the first statement of Proposition 4.1.

So assume now $-(\mathbf{t}, \mathbf{n}) \in C$. As before, it suffices to consider a neighborhood of a given point $x$ in the compact set $Y_{C}$. Let $\eta>0$ and let $B=B_{\eta}^{U^{+}}(e) B_{\eta}^{U^{-}}(e) B_{\eta}^{C_{G}(a)}(e)$, where $C_{G}(a)$ is the centralizer of $a$ in $G$. Note that $B \subset G$ is the neighborhood of $e$, and that $B \subset B_{\delta}(e)$ for small enough $\eta$. Moreover, the metric restricted to $B$ is Lipshitz equivalent to the product metric on $B_{\eta}^{U^{+}}(e) \times B_{\eta}^{U^{-}}(e) \times B_{\eta}^{C_{G}(a)}(e)$. If the last statement of Proposition 4.1 fails for the intersection $Y_{C} \cap(B x)$, then the image $I_{B, x}$ of the projection of $E_{B, x}=\left\{u \in B: u x \in Y_{C}\right\}$ to $B_{\eta}^{U^{+}}(e) \times B_{\eta}^{U^{-}}(e)$ has positive box dimension. We show that this implies that $\mathrm{h}_{\text {top }}(\beta)>0$. If $u=u^{+} u^{-} u^{0}, v=$ $v^{+} v^{-} v^{0} \in E_{B, x}$ (with $u^{+}, u^{-}, \ldots$ as in the definition of $B$ ) are such that $d\left(u^{+} u^{-}, v^{+} v^{-}\right)>\lambda^{-n}$, then either $d\left(u^{+}, v^{+}\right)>\lambda^{-n} / C$ or $d\left(u^{-}, v^{-}\right)>\lambda^{-n} / C$ for some absolute constant $C$ (coming from the Lipshitz constants between the two metrics on $B$ ). In the former case, we find, for a small enough $\epsilon>0$, just as in the second paragraph of the proof, a $j$ with $0 \leqslant j<n, \epsilon<d\left(a^{j} u^{+} a^{-j}, a^{j} v^{+} a^{-j}\right)<\delta$ and $d\left(a^{j} u^{-} u^{0} a^{-j}, a^{j} v^{-} v^{0} a^{-j}\right)<c \eta$ for some absolute constant $c$. For $\epsilon^{\prime}=\epsilon / 2$ this implies, for small enough $\eta$, that $\epsilon^{\prime}<d\left(a^{j} u a^{-j}, a^{j} v a^{-j}\right)<2 \delta$. The second case leads to a similar estimate for some $j$ with $-n<j \leqslant 0$. Using the invariance of $Y_{C}$ under $\alpha^{-(\mathbf{t}, \mathbf{n})}$ this shows that $s_{\epsilon^{\prime}, 2 n-1}(\beta) \geqslant s_{\lambda^{-n}}\left(I_{B, x}\right)$. As above, this gives the desired result.

We can link Lemma 4.2 with the measure-theoretic entropy by the variational principle (see [Wal82, Theorem 8.6]): for any continuous map $\beta: Y \rightarrow Y$ of a compact metric space $Y$, its topological entropy is the supremum

$$
\mathrm{h}_{\text {top }}(\beta)=\sup _{\nu} \mathrm{h}_{\nu}(\beta)
$$

over all measure-theoretic entropies with respect to $\beta$-invariant probability measures $\nu$ on $Y$.

Proof of Proposition 4.1. By Lemma 4.2 it suffices to assume that $\mathrm{h}_{\text {top }}(\beta)>0$ and to conclude with a contradiction.

By the variational principle (4.1) there exists a $\beta$-invariant measure $\nu$ (supported on the compact set $Y_{C}$ where $\beta$ is defined) such that $\mathrm{h}_{\nu}(\beta)$ is as close to $\mathrm{h}_{\text {top }}(\beta)$ as we want. All we need is $\mathrm{h}_{\nu}(\beta)>0$. Since the entropy of $\beta$ with respect to a non-ergodic measure $\nu$ can be calculated as an integral of the entropies of $\beta$ with respect to the ergodic components of $\nu$, we can assume that $\nu$ is $\beta$-ergodic.

There is no reason for $\nu$ to be $\alpha$-invariant, so we need to go through the following averaging procedure. Let $\lambda$ denote the Haar measure on $\mathbb{R}^{k} \times \mathbb{Z}^{\ell}$ and let $Q_{N}=[-N, N]^{k} \times\{-N, \ldots, N\}^{\ell}$ be 
a large box in $\mathbb{R}^{k} \times \mathbb{Z}^{l}$. Define the sequence of measures

$$
\nu_{N}=\frac{1}{\lambda\left(Q_{N} \cap C\right)} \int_{Q_{N} \cap C}\left(\alpha^{(\mathbf{s}, \mathbf{m})}\right)_{*} \nu d \lambda(\mathbf{s}, \mathbf{m})
$$

on $Y_{C}$. Then the definition of the cone implies for a given $(\mathbf{s}, \mathbf{m})$ that for large $N$ the measure $\left(\alpha^{(\mathbf{s}, \mathbf{m})}\right)_{*} \nu_{N}$ is close (in strong or weak* topology) to $\nu_{N}$. Therefore, every weak ${ }^{*}$ accumulation point $\bar{\nu}$ of the sequence $\nu_{N}$ will be an $\alpha$-invariant probability measure on $Y_{C}$.

In many different settings it is well known (see [Wal82, Theorem 8.2] and [New89, Theorem 4.1]) that the measure-theoretic entropy depends upper semi-continuously on the measure (using the weak* topology). These theorems do not quite apply to the proof here, but it is easy to adapt the proof of [EKL06, Corollary 10.3] to $\beta$. By definition of $\nu_{N}$ in (4.2) the ergodic components of $\nu_{N}$ with respect to $\beta$ are all of the form $\left(\alpha^{(\mathbf{s}, \mathbf{m})}\right)_{*} \nu$. Therefore, $\mathrm{h}_{\nu_{N}}(\beta)=\mathrm{h}_{\nu}(\beta)$ for all $N$, and upper semi-continuity implies that

$$
\mathrm{h}_{\bar{\nu}}(\beta) \geqslant \mathrm{h}_{\nu}(\beta)>0 \text {. }
$$

Again because entropy can be expressed as an integral, this time over all ergodic components $\mu$ of $\bar{\nu}$ with respect to $\alpha$, there exists an $\alpha$-invariant and ergodic measure $\mu$ that is supported by $Y$ with $\mathrm{h}_{\mu}\left(\alpha^{(\mathbf{t}, \mathbf{n})}\right)>0$. This is a contradiction to the assumption of Proposition 4.1.

\section{Conclusion}

\subsection{Completion of the proof of Theorem $\mathbf{1 . 2}$}

Proof of Theorem 1.2. We first consider the case $v \in \mathbb{Z}_{p}$ and define

$$
E_{v}=\left\{u \in \mathbb{R}: \liminf _{q \rightarrow \infty, q_{0} \rightarrow \infty}|q| \cdot\left|q u-q_{0}\right| \cdot\left|q v-q_{0}\right|_{p}>0\right\} .
$$

Here Proposition 2.2 shows $u \in E_{v}$ if and only if $\alpha^{C} x_{u, v}$ is bounded, i.e. $\alpha^{C} x_{u, v} \subset K_{\delta}$ for some $\delta>0$, where $K_{\delta}$ are the compact subsets defined in $\S 2.1$. Therefore,

$$
E_{v}=\bigcup_{\delta>0} E_{v}(\delta)
$$

where

$$
E_{v}(\delta)=\left\{u \in[-1 / \delta, 1 / \delta]: \alpha^{C}\left(\left(\begin{array}{ll}
1 & \\
u & 1
\end{array}\right),\left(\begin{array}{ll}
1 & \\
& 1
\end{array}\right)\right) x_{0, v} \subset K_{\delta}\right\} .
$$

By Corollary 3.2, $K_{\delta} \neq X$ does not support any $\alpha$-invariant and ergodic probability measure $\mu$ with $\mathrm{h}_{\mu}\left(\alpha^{(1,0)}\right)>0$. It is easy to check that

$$
U=\left\{\left(\left(\begin{array}{ll}
1 & \\
u & 1
\end{array}\right),\left(\begin{array}{ll}
1 & \\
& 1
\end{array}\right)\right): u \in \mathbb{R}\right\}
$$

is the horospherical unstable subgroup corresponding to $\alpha^{(1,0)}$. Therefore, $E_{v}(\delta)$ has box dimension zero by Proposition 4.1, and so $E_{v}$ is a countable union of such sets as claimed in Theorem 1.2. The fact that $E_{v}$ has Hausdorff dimension zero follows from this: the Hausdorff dimension is always less than or equal to the box dimension, and the Hausdorff dimension of a countable union is the supremum of the Hausdorff dimensions of the original sets.

Now let $v \in \mathbb{Q}_{p}$ and fix some $n \geqslant 0$ with $p^{n} v \in \mathbb{Z}_{p}$. Using the definition only, it is straightforward to check that $p^{n} E_{v} \subset E_{p^{n} v}$. Since the latter satisfies the conclusion of the theorem, so does $E_{v}$.

\subsection{Furstenberg's theorem and Theorem 1.3}

Let $\mathbb{T}=\mathbb{R} / \mathbb{Z}$ and denote by $\times p$ the map $x \mapsto p x$ (modulo one). Recall that a closed subset $F \subset \mathbb{T}$ is $\times p$-invariant if $\times p(F) \subset F$. 


\section{EinsiedleR AND D. Kleinbock}

Theorem 5.1 (Furstenberg [Fur67, Theorem IV.1]). Let $p_{1}, p_{2}$ be two distinct primes. A closed subset $F \subset \mathbb{T}$ that is $\times p_{1}, \times p_{2}$-invariant is either a finite set of rational points or equal to $\mathbb{T}$.

Proof of Theorem 1.3. For two primes $p_{1}, p_{2}$, and $\delta>0$, consider the set

$$
F_{\delta}=\left\{u \in \mathbb{R}: \inf _{q \in \mathbb{N}}|q| \cdot|q|_{p_{1}} \cdot|q|_{p_{2}} \cdot\langle q u\rangle \geqslant \delta\right\} / \mathbb{Z} .
$$

From the definition it is clear that $F_{\delta}$ is closed. We claim it is also $\times p_{1}, \times p_{2}$-invariant. Indeed, let $u \in F_{\delta}$ and $q \in \mathbb{N}$, then

$$
|q| \cdot|q|_{p_{1}} \cdot|q|_{p_{2}} \cdot\left\langle q p_{1} u\right\rangle=\left|q p_{1}\right| \cdot\left|q p_{1}\right|_{p_{1}} \cdot\left|q p_{1}\right|_{p_{2}} \cdot\left\langle q p_{1} u\right\rangle \geqslant \delta .
$$

Since $q \in \mathbb{N}$ was arbitrary, $p_{1} u \in F_{\delta}$. Clearly, $F_{\delta}$ does not contain any rational numbers. By Theorem $5.1, F_{\delta}$ must be empty, which shows that for every $u \in \mathbb{R}$ there exists $q \in \mathbb{N}$ with

$$
|q| \cdot|q|_{p_{1}} \cdot|q|_{p_{2}} \cdot\langle q u\rangle<\delta
$$

If $u$ is rational there is nothing to prove. Otherwise, it is clear that for small $\delta>0$ the natural number $q$ as above needs to be rather large. Therefore, (1.9) is satisfied and the theorem follows.

\section{$5.3 \mathcal{D}$-adic valuations}

Following [DT04], let us consider an increasing sequence $\mathcal{D}=\left(r_{n}\right)_{n \in \mathbb{Z}_{+}}$of positive integers with $r_{0}=1$ and $r_{n+1} \in r_{n} \mathbb{Z}$ for each $n$, and define the $\mathcal{D}$-adic pseudo-valuation

$$
|q|_{\mathcal{D}} \stackrel{\text { def }}{=} \inf \left\{1 / r_{n}: q \in r_{n} \mathbb{Z}\right\} .
$$

Clearly $|\cdot|_{\mathcal{D}}=|\cdot|_{p}$ for $\mathcal{D}=\left\{p^{n}\right\}$, in which case $|\cdot|_{\mathcal{D}}$ is multiplicative. De Mathan and Teulié showed that whether or not the equality

$$
\liminf _{q \rightarrow \infty}|q| \cdot|q|_{\mathcal{D}} \cdot\langle q u\rangle=0
$$

holds is equivalent to the digits in the continued fraction expansions for $r_{n} u$ not having a uniform bound. They also proved that (5.1) holds when $u$ is quadratic irrational and

$$
\text { ratios } r_{n+1} / r_{n} \text { are uniformly bounded, }
$$

and asked whether it holds for all real numbers $u$.

It seems tempting to attack this problem using the methods of the present paper. Unfortunately, for general $\mathcal{D}$, even satisfying (5.2), we do not know how to translate (5.1) into the language of group actions. However, the special case $\mathcal{D}=\left\{a^{n}\right\}$, where $a$ is not necessarily prime, happens to be amenable to our technique and we can assert that for this choice of $\mathcal{D}$ the set of exceptions to (5.1) has Hausdorff dimension zero. Further, if we denote by $S$ the set $\left\{p_{1}, \ldots, p_{\ell}\right\}$ of primes dividing $a$, the completion of $\mathbb{Q}$ with respect to $|\cdot|_{\mathcal{D}}$ is bi-Lipschitz equivalent to $\mathbb{Q}_{S} \stackrel{\text { def }}{=} \prod_{i} \mathbb{Q}_{p_{i}}$ (where the metric on $\mathbb{Q}_{p_{i}}$ is scaled by the exponent with which $p_{i}$ occurs in $a$ ). Then it can be shown that for any $v \in \mathbb{Q}_{S}$, the set of $u \in \mathbb{R}$ which do not satisfy

$$
\liminf _{q \rightarrow \infty, q_{0} \rightarrow \infty}|q| \cdot\left|q u-q_{0}\right| \cdot\left|q v-q_{0}\right|_{\mathcal{D}}=0
$$

is a countable union of sets with box dimension zero.

The proof uses dynamics on $X=G / \Gamma$, where

$$
G=\mathrm{SL}(2, \mathbb{R}) \times \mathrm{SL}\left(2, \mathbb{Q}_{S}\right)=\mathrm{SL}(2, \mathbb{R}) \times \prod_{i} \mathrm{SL}\left(2, \mathbb{Q}_{p_{i}}\right),
$$

and $\Gamma$ is $\operatorname{SL}\left(2, \mathbb{Z}\left[\frac{1}{p_{1}}, \ldots, \frac{1}{p_{\ell}}\right]\right)$ diagonally embedded in $G$. The action considered is left multiplication 
by

$$
\left(\left(\begin{array}{cc}
e^{-t} & \\
& e^{t}
\end{array}\right),\left(\begin{array}{cc}
a^{n} & \\
& a^{-n}
\end{array}\right)\right) \text { for }(t, n) \in \mathbb{R} \times \mathbb{Z},
$$

where $\left(a^{n} a^{-n}\right)$ is understood to be diagonally imbedded in $\operatorname{SL}\left(2, \mathbb{Q}_{S}\right)$. One can prove that $u \neq 0$ (respectively, $u \neq 0$ and $v \in \prod_{i} \mathrm{SL}\left(2, \mathbb{Z}_{p_{i}}\right)$ ) fail (5.1) (respectively, (5.3)) if and only if certain elements of $X$ as above have bounded orbits relative to certain cones in $\mathbb{R} \times \mathbb{Z}$. Then one applies Theorem 3.1 with $L=\operatorname{SL}\left(2, \mathbb{Q}_{S}\right)$.

\subsection{Extending Theorem 1.2}

Theorem 1.2 can be strengthened in another direction. Namely, the following is true.

Theorem 5.2. The set of $(u, v) \in \mathbb{R} \times \mathbb{Q}_{p}$ which do not satisfy

$$
\liminf _{q \rightarrow \infty, q_{0} \rightarrow \infty}|q| \cdot\left|q u-q_{0}\right| \cdot\left|q v-q_{0}\right|_{p}=0
$$

is at most a countable union of sets with box dimension zero.

A stronger version of Theorem 3.1 is needed for the above, namely that the Haar measure $m_{X}$ is the only $\alpha$-invariant and ergodic probability measure with $\mathrm{h}_{\mu}\left(\alpha^{(t, n)}\right)>0$ for some $(t, n) \in \mathbb{R} \times \mathbb{Z}$. The case $(t, n)=(0,1)$ opposite to Theorem 3.1 where $(t, n)=(1,0)$ can be proven with the same method as used in the main theorem of [Lin06]. However, we claim that

$$
\mathrm{h}_{\mu}\left(\alpha^{(t, n)}\right)=\mathrm{h}_{\mu}\left(\alpha^{(t, 0)}\right)+\mathrm{h}_{\mu}\left(\alpha^{(0, n)}\right),
$$

and so the above two cases give the complete answer for measures with positive entropy. This additivity of entropy follows from [EK05], since for a general $(t, n)$, the stable horospherical subgroup $U$ is the product of its real subgroup $U_{\infty}$ and its $p$-adic subgroup $U_{p}$, and the Lyapunov weights that describe the contraction (respectively, expansion) rate for $U_{\infty}$ (respectively, $U_{p}$ ) are linearly independent (indeed, $\alpha^{(1,0)}$ commutes with the $p$-adic group $U_{p}$ but not with $U_{\infty}$, and similarly for $\left.\alpha^{(0,1)}\right)$. By [EK05, Theorem 8.4] this shows that the conditional measures for $U$ are the product measures of the conditional measures for $U_{\infty}$ and $U_{p}$. By [EK05, Proposition 9.4] these conditional measures determine entropy which implies the above.

A more general measure rigidity theorem, covering this as well as other more general situations, is an ongoing joint work of Lindenstrauss and the first named author.

\section{ACKNOWLEDGEMENTS}

The authors are grateful to University of Washington and Brandeis University for arranging their mutual visits, and to the Max Planck Institute for its hospitality during July 2004. Thanks are also due to Yann Bugeaud, Elon Lindenstrauss and George Tomanov for helpful discussions. This research was partially supported by NSF grants 0239463,0400587 and 0509350.

\section{REFERENCES}

CS55 J. Cassels and H. Swinnerton-Dyer, On the product of three homogeneous linear forms and the indefinite ternary quadratic forms, Philos. Trans. Roy. Soc. London Ser. A 248 (1955), 73-96; MR $17,14 f$.

DT04 B. de Mathan and O. Teulié, Problèmes diophantiens simultanés, Monatsh. Math. 143 (2004), 229-245.

EK03 M. Einsiedler and A. Katok, Invariant measures on $G / \Gamma$ for split simple Lie-groups $G$, Comm. Pure Appl. Math. 56 (2003), 1184-1221. 


\section{MeAsure Rigidity AND $p$-ADIC LitTlewOod-TyPe PROBlems}

EK05 M. Einsiedler and A. Katok, Rigidity of measures - the high entropy case, and non-commuting foliations, Israel J. Math. 148 (2005), 169-238.

EKL06 M. Einsiedler, A. Katok and E. Lindenstrauss, Invariant measures and the set of exceptions to Littlewood's conjecture, Ann. of Math. (2) 164 (2006), 513-560.

Fur67 H. Furstenberg, Disjointness in ergodic theory, minimal sets, and a problem in Diophantine approximation, Math. Systems Theory 1 (1967), 1-49.

Jac63 K. Jacobs, Ergodic decomposition of the Kolmogoroff-Sinai invariant, in Proc. int. symp. on ergodic theory, Tulane University, New Orleans, LA, 1961 (Academic Press, New York, 1963), 173-190.

KK01 B. Kalinin and A. Katok, Invariant measures for actions of higher rank abelian groups, in Smooth ergodic theory and its applications, Seattle, WA, 1999 (American Mathematical Society, Providence, RI, 2001), 593-637.

KS05 B. Kalinin and R. Spatzier, Rigidity of the measurable structure for algebraic actions of higher-rank Abelian groups, Ergodic Theory Dynam. Systems 25 (2005), 175-200.

KT D. Kleinbock and G. Tomanov, Flows on S-arithmetic homogeneous spaces and applications to metric diophantine approximation, Comment. Math. Helv., to appear.

Kne65 M. Kneser, Starke Approximation in algebraischen Gruppen. I, J. reine angew. Math. 218 (1965), 190-203.

Lin06 E. Lindenstrauss, Invariant measures and arithmetic quantum unique ergodicity, Ann. of Math. (2) 163 (2006), 165-219.

Mar97 G. Margulis, Oppenheim conjecture, in Fields Medallists' lectures, World Scientific Series in 20th Century Mathematics, vol. 5 (World Scientific, River Edge, NJ, 1997), 272-327.

Mar00 G. Margulis, Problems and conjectures in rigidity theory, in Mathematics: frontiers and perspectives (American Mathematical Society, Providence, RI, 2000), 161-174.

MT94 G. Margulis and G. Tomanov, Invariant measures for actions of unipotent groups over local fields on homogeneous spaces, Invent. Math. 116 (1994), 347-392.

New89 S. E. Newhouse, Continuity properties of entropy, Ann. of Math. (2) 129 (1989), 215-235.

Rag72 M. S. Raghunathan, Discrete subgroups of Lie groups (Springer, Berlin, 1972).

Rat91 M. Ratner, On Raghunathan's measure conjecture, Ann. of Math. (2) 134 (1991), 545-607.

Rat95 M. Ratner, Raghunathan's conjectures for Cartesian products of real and p-adic Lie groups, Duke Math. J. 77 (1995), 275-382.

Sta00 A. N. Starkov, Dynamical systems on homogeneous spaces, Translations of Mathematical Monographs, vol. 190 (American Mathematical Society, Providence, RI, 2000).

Tom00 G. Tomanov, Orbits on homogeneous spaces of arithmetic origin and approximations, in Analysis on homogeneous spaces and representation theory of Lie groups, Okayama-Kyoto, 1997, Advanced Studies in Pure Mathematics, vol. 26 (Mathematical Society of Japan, Tokyo, 2000), 265-297.

Var63 V. S. Varadarajan, Groups of automorphisms of Borel spaces, Trans. Amer. Math. Soc. 109 (1963), 191-220.

Wal82 P. Walters, An introduction to ergodic theory, Graduate Texts in Mathematics, vol. 79 (Springer, New York, 1982).

Manfred Einsiedler einsiedler.1@osu.edu

Department of Mathematics, The Ohio State University, 100 Math Tower, 231 West 18th Avenue, Columbus, OH 43210-1174, USA

Dmitry Kleinbock kleinboc@brandeis.edu

Department of Mathematics, MS 050, Brandeis University, PO Box 9110, Waltham, MA 02454, USA 